\title{
Initiative to Improve the Health Outcomes of Those at Risk of Perinatal Depression: Referral Characteristics and Psychosocial Determinants
}

\author{
Mona Asghari-Fard' ${ }^{1}$, Ursula Hopper ${ }^{2}$, My Trinh Ha ${ }^{1}$, Valsamma Eapen ${ }^{3,4 *}$ \\ ${ }^{1}$ Academic Unit of Child Psychiatry, South Western Sydney, Liverpool Hospital, Sydney, Australia \\ ${ }^{2}$ Perinatal Mental Health Service, Liverpool Hospital, Sydney, Australia \\ ${ }^{3}$ Academic Unit of Child Psychiatry, South West Sydney (AUCS) ICAMHS, Mental Health Centre, \\ Liverpool Hospital, Sydney, Australia \\ ${ }^{4}$ School of Psychiatry \& Ingham Institute, University of New South Wales, Sydney, Australia \\ Email: *v.eapen@unsw.edu.au
}

Received 30 May 2016; accepted 9 July 2016; published 12 July 2016

Copyright (C) 2016 by authors and Scientific Research Publishing Inc.

This work is licensed under the Creative Commons Attribution International License (CC BY).

http://creativecommons.org/licenses/by/4.0/

(c) (i) Open Access

\begin{abstract}
In Australia, perinatal depression affects 15\% - 20\% of pregnant women. Depression does not go away on its own, getting help at early stages shown to be effective in treating antenatal depression. Aim of this study is to assess and describe the screening of women through the antenatal clinic and measure the outcome of services provided (such as counselling, social assistance) for those at risk of depression, in a general hospital setting in an ethnically diverse part of Sydney, Australia. Data from 193 women were obtained through accessing the psychosocial and screening assessments completed at the antenatal clinic between 2007 and 2008. Data regarding patients' psychosocial characteristics, referrals and interventions were also gathered from hospital records. Data revealed that $60.4 \%$ of women screened scored $\geq 10$ on the Edinburgh Postnatal Depression Scale (EDPS) which is indicative of significant depressive symptomatology. Of these women, $39.4 \%$ went on to receive a formal diagnosis. Women who indicated that they had planned their pregnancies $(47.2 \%)$ were significantly less likely to report having major worries and stressors over the last 12 months $(p<0.05)$ in comparison to those who indicated that their pregnancies were unplanned. Data showed while screening methods are effective, regrettably a high proportion of women, despite presenting with "at risk" symptomatology levels, do not engage in intervention programs. Further research is required to explore the barriers in accessing both screening and intervention services (particularly in a culturally diverse area such as this), and how services can improve processes and patient participation.
\end{abstract}

*Corresponding author.

How to cite this paper: Asghari-Fard, M., Hopper, U., Ha, M.T. and Eapen, V. (2016) Initiative to Improve the Health Outcomes of Those at Risk of Perinatal Depression: Referral Characteristics and Psychosocial Determinants. Open Journal of Obstetrics and Gynecology, 6, 463-472. http://dx.doi.org/10.4236/ojog.2016.68062 
Keywords

\section{Antenatal Depression, Routine Screening, Early Identification}

\section{Introduction}

Depression has been recognised as a significant health burden both in Australia and around the world with the World Health Organisation (WHO) stating that it is the leading cause of disability worldwide [1]. In Australia perinatal affects 15 - 20 percent of Australian women [2]. The perinatal mental health remains as a major public health concern around the world [3]. Although much research has focused on effective diagnosis and treatment for unipolar depression and increasingly postnatal depression, limited research has focused on depression that occurs during the antenatal period. Few studies have examined the occurrence of depression from pregnancy, but available evidence indicates that depression during pregnancy is as prevalent as during the postpartum period [4]. Furthermore, research has shown that a large proportion of women who have received a diagnosis of depression postnatally, report having depressive symptoms that began much earlier during the antenatal period [5]; in fact it has been shown that $50 \%-70 \%$ of postpartum depression and anxiety begin in pregnancy [6]. In an Australian study, Matthey, Barnett [7] showed that $42.1 \%$ of women, who met criteria for antenatal depression, experienced a recurrence of the depression during the postnatal period thus highlighting the significance of antenatal depression as a risk factor for postpartum depression. A recent Western European study among ethnic minority groups found that the prevalence of depression in pregnancy was higher in women from the Middle East and South Asia with rates of 19.5\% (12.19 - 26.81) in Middle Easterners and 17.5\% (12.08 - 22.92) among South Asians as compared with 11.3\% (6.09 - 16.51) in other groups and 8.6\% (95\% CI: 5.45 - 11.75) in Western Europeans [8].

The impacts of antenatal and postnatal depression are not exclusive to the woman diagnosed. In fact the development and onset of perinatal depression can have detrimental effects on the entire family unit [9]-[13]. More specifically, these disorders can impact a woman's ability to perform daily activities, maintain relationships, and form quality attachments with her infant [9] and parent sensitively [14]. In addition, maternal depression can also place the infant at risk of broader developmental issues [14] [15] and is a significant risk factor for poorer psychosocial outcomes for the mother and the infant [16].

Given the far reaching and significant effect of maternal depression (both perinatal and postnatal) and despite clinicians' growing recognition and identification of depression during the perinatal period, studies show that pregnancy related depressive disorders may still go unrecognised [17]-[19] and thus untreated. Some research has suggested that misdiagnosis (or no diagnosis) may occur during the antenatal period due to the overlap of common pregnancy related symptoms and depressive symptoms (e.g., fatigue) [9] [14]. Rates of misdiagnosis have been reported as being as high as $50 \%$ (see Lien, 2007). A report commissioned in the UK to review practises and antenatal screening processes for depression determined that the existing screening processes were unacceptable, and highlighted that general practitioners and primary care providers were diagnosing antenatal depression late or misdiagnosing women altogether [11]. In Australia, a study assessed midwives' levels of knowledge of antenatal and postpartum depression. Their findings revealed that $70.6 \%$ of midwives were unable to identify the risk factors of antenatal depression and that $98.3 \%$ of respondents underestimated the percentage of suicide attempts made by women with antenatal depression [20].

There has been a recent focus within health systems around the world to develop processes and guidelines that enable effective early identification and intervention [21] to improve outcomes for women and children [14]. In the US for example, public health initiatives have focused on the development of national guidelines that ensure reliable and systematic screening processes through training and educating primary health care providers. Although the various training and education programs have increased screening rates and thus rates of detection, most agencies still report that there a need for improvement.

Similarly, in recognising the importance of accurate and early identification of women who are at risk of developing perinatal depression in Australia, the Beyond Blue National Postnatal Depression Program was established across five states with the aim of evaluating screening for perinatal depression. The development of this program led to the establishment of the National Perinatal Depression Initiative (NPDI) which aimed to foster collaboration between State and Territory Governments and the Federal Government to develop materials and 
training to support professional practice, facilitating the linking of all services involved in the care of perinatal women, and increasing community and health professional awareness [22].

Despite the efforts to improve education and support, screening processes in the first instance can vary and rely heavily on self-report measures. One of the most commonly used and widely accepted screening instruments is the Edinburgh Postnatal Depression Scale (EPDS; [23]). Although the instrument was developed primarily to assess symptomatology postpartum, recent studies have validated its use within the antenatal context [24] and for use with women from different cultural backgrounds [25].

Given the importance of providing effective screening processes, to improve diagnosis and intervention strategies and thus improve psychosocial outcomes for women and their families, it is vital that there is a continuous evaluation of health services [26]. Hence in order to evaluate whether our health services meet the needs of our clients, this study aimed to identify and describe.

1) The prevalence of women at risk of depression who were identified during pregnancy as part of the antenatal psychosocial screening program.

2) The socio demographic and psychosocial characteristics of women who screened positive on the EPDS as scoring $\geq 10$.

3) The nature of the referral process and the referral outcomes for those women who were identified as being "at risk" of depression or having clinical levels of depression as identified on the EPDS.

This paper will also discuss and explore current screening limitations and service improvements within the antenatal unit at hand.

\section{Method}

This evaluation study examined clinical data that was collected from women as part of routine admission procedures in a Hospital antenatal unit between 2007 and 2008. Data collected from women who had been referred to the Perinatal Mental Health Service (PIMHS) in the same period were also examined. Ethics approval to access patients' data from the service data bases was obtained from the Institutional Ethics Committee.

Women attending for their initial appointments at the antenatal clinic routinely complete a socio demographic questionnaire and the EPDS is an important element of this screening process.

Women who are found to be either at risk of or having depression as evidenced by a score of $\geq 10$ on the EPDS are referred for assessment and treatment.

\subsection{Referral Process to Perinatal and Infant Mental Health Services (PIMHS)}

In Australian health systems, antenatal clinics work closely with Perinatal and Infant Mental Health Services (PIMHS) to provide an early identification and intervention service aimed at ensuring optimal psychological, social and physical health of pregnant women. As mentioned above women who are found to be either at risk would be referred to PIMHS. Following their referral, all patients flagged by the midwives as being at risk, based on these measures, are discussed at the weekly Perinatal Referral Meeting involving social workers, mental health, drug and alcohol, midwifery, women's health, child and family health professionals, and other not-for profit community services. From these cases, those deemed to need active intervention by the mental health team are referred to the PIMHS team. Once a referral is received by the PIMHS team, those in need of pharmacological treatment are seen by the team psychiatrist or referred to their family GPs. For other patients (that do not require pharmacological interventions), a management plan is developed in consultation with the multidisciplinary team. Management plans may include individual counselling/psychological therapy or referral to community agencies for support services.

\subsection{Screening Measures}

As a part of routine referral procedures, all women who presented or were referred to the PIMHS unit provided socio demographic data including date of birth, age, country of birth, marital status, employment status, language spoken and residential suburb. In addition to the socio-demographic data, patients' initial Edinburgh Postnatal Depression Scale scores EPDS; [23] and any subsequent EPDS scores were also accessed for analysis. Patients' perinatal psychosocial risk and details regarding referrals, interventions provided and patient outcomes data was also collected and examined. 


\subsubsection{The Antenatal Psychosocial Assessment (APA)}

The Antenatal Psychosocial Assessment (APA) developed as a part of the NSW Health SAFE START guidelines [27] is a 23 item instrument which assesses the patient's level of support (social and emotional), mental health history, and adverse life events (including whether they have experienced childhood abuse). Patients attending the antenatal clinic are asked to complete this questionnaire as a part of their routine assessment [28]. Data ascertained from this assessment were included in the analysis.

\subsubsection{The Edinburgh Postnatal Depression Scale (EPDS)}

The Edinburgh Postnatal Depression Scale (EPDS; Cox et al., 1987) comprises of 10 self-report statements where respondents indicate the degree to which they have felt symptoms within the last 7 days. Responses are made on a 4 point Likert scale. Higher total scores on the EDPS indicate a more severe symptom profile. Scores of 9 or less are defined as subclinical and nonsymptomatic. Although EPDS clinical cut off scores have varied in previous research most researchers indicate that a threshold value of 10 yields the best sensitivity at $100 \%$ but a specificity of $87 \%$ resulting in many false positives and suggest a need for follow up and ongoing monitoring [29]. Scores above 12 however are taken to suggest a very high likelihood of depression and requirement of further assessment with a view of intervention (Cox and Holden, 1987). Research studies have shown that the EPDS has satisfactory to high levels of sensitivity and specificity for use with women and can detect changes in the severity of depressive symptoms over time [23] [30] [31].

\subsection{Data Analysis}

Data was analysed using SPSS version 22.0. Descriptive statistics are presented in Table 1. More specifically, two-way chi-square analyses were performed to determine whether there were any significant associations between socio-demographic details and APA responses. One way ANOVA's and paired t-tests were also conducted to determine whether there were significant differences between groups.

Table 1. Descriptive information and frequencies (\% of respondents) obtained from the antenatal psychosocial assessment completed by participants $(\mathrm{n}=193)$.

\begin{tabular}{|c|c|}
\hline Basic demographics and information & $\%$ of women \\
\hline Age & $\mathrm{M}=31.58, \mathrm{SD}=5.58$ \\
\hline \multicolumn{2}{|l|}{ Language Spoken at Home } \\
\hline English & $44 \%$ \\
\hline Other & $56 \%$ \\
\hline Pregnancy was planned & $47.2 \%$ \\
\hline \multicolumn{2}{|l|}{ Support } \\
\hline Has emotional support from partner & $81 \%$ \\
\hline Will have practical support following birth of child & $88.6 \%$ \\
\hline Someone to talk to about worries and feelings & $86.4 \%$ \\
\hline \multicolumn{2}{|l|}{ Mental Health Factors } \\
\hline Has had major stressors or worries in the last 12 months & $49.6 \%$ \\
\hline Worries if things get messy or out of place & $34.8 \%$ \\
\hline Have had treatment for emotional/mental health problems & $48.9 \%$ \\
\hline Have experienced abuse as a child & $27.7 \%$ \\
\hline Has had thoughts of self harm (based on the EPDS item) & $14.5 \%$ \\
\hline \multicolumn{2}{|l|}{ Intervention/Referral } \\
\hline Referral made & $67 \%$ \\
\hline Referral declined & $5.6 \%$ \\
\hline
\end{tabular}




\subsection{Ethical Considerations}

Health care quality improvement research (QIR) is considered minimal risk projects because researchers are not introducing an intervention, simply accessing collected data by clinician. This evaluation study submitted low negligible risk (LNR) application and gained approval for the study.

\section{Results}

Data from 193 women who presented at the antenatal and PIMHS units within a SWSLHD public hospital between the years 2007 and 2008 was accessed using electronic medical records and PIMHS register of referrals.

\subsection{Descriptive}

Data retrieved from the registers revealed that the mean age of women who presented at the hospital was 31 years $(S D=5.6)$. Forty four percent of the women who attended and provided data indicated that they were 235 reborn in Australia, and 44\% indicated that English was their first language. Other languages that were identified were Croatian and Khmer.

\subsection{Antenatal Psychosocial Assessment Findings}

Data indicated that less than half of the women who attended had planned their pregnancies (47.2\%). Eighty one percent of women felt that their partner was emotionally supportive of them and $86.4 \%$ of women felt that they had someone in their lives that they could speak to about their feelings and worries. Moreover, $88.6 \%$ of women who completed the assessment questionnaires indicated that they are able to get partner and/or family support following the birth of their child. $7.7 \%$ of women indicated that they felt that they would not be supported or would receive minimal support.

In terms of respondents' psychosocial wellbeing, $49.6 \%$ of women reported that they had major worries or stress in the last 12 months, while almost a quarter of responding women indicated that they had felt depressed or anxious for a period of two or more weeks (24.2\%) and that some of these episodes were related to their pregnancy (27.3\%). $48.9 \%$ of women reported that they had received treatment for a mental health problem in the past and $14.5 \%$ of women indicated that they had thoughts of self-harm.

\subsection{Edinburgh Scale}

Data also revealed that of $60.4 \%$ of the overall sample scored $\geq 10$ on the EPDS which is indicative of high and significant depressive symptomatology. 53.8\% of women scored above 12 on their initial EPDS. Of the women who completed the assessment, $27.7 \%$ indicated that they had experienced some form of child abuse.

\subsection{Association between EDS Sore and Other Variables}

Of the women who scored more than 10 on their initial EPDS ( $n=55, m=15.47, \mathrm{SD}=4.38$ ), 39.4\% had been formally diagnosed with either depression or anxiety, $15.2 \%$ with comorbid depression and anxiety and records for $18.2 \%$ of the women revealed that they had an existing mental health illness. Over $1 / 3$ of women who scored 10 or more on their initial EPDS indicated that they had experienced some form of abuse as a child (see Table 1). Close to $67 \%$ of these women were referred onto receive further psychosocial treatment/assessment, $5.6 \%$ of these women declined a referral. Similar percentages of women who scored 12+ on their initial EPDS were referred on (63.9\%). Of those who were referred to PIMHS for intervention, around $72.8 \%$ were diagnosed by professionals as having either depression or anxiety (31.4\%), comorbid depression and anxiety (20\%), or an existing mental health issue (20\%).

Two-way chi-square analyses revealed that there was a significant relationship between whether the pregnancy was planned and experiencing major worries or stresses over the last 12 months $\left(X^{2}(1)=10.792, p=0.001\right)$, indicating that those who had unplanned pregnancies had been more likely to report that they had significant worries or stressors in the last 12 months. Analyses indicated that there was not a significant relationship between whether the pregnancy was planned and whether patients experienced anxiety/depression for a period of 2 weeks or more. 
Chi-square analyses also showed that there was no significant association between those women who felt that they had support from either their partner or elsewhere and whether or not they experienced major worries/stressors in the last 12 months $\left(\mathrm{X}^{2}(1)=0.618, \mathrm{p}>0.05\right)$.

Significant associations were however found between childhood abuse and reported stress and anxiety over the last 12 months $\left(\mathrm{X}^{2}(1)=8.483\right.$, $\left.\mathrm{p}=0.004\right)$ indicating that for those women who reported that they had experienced some form of abuse as a child, were more likely to report that they had experienced major stress/worries in the last 12 months. There were no significant associations however between the experience of childhood abuse and whether women had experienced depression or anxiety for a period of two weeks or more. Moreover, a one-way ANOVA revealed that there was no significant difference in initial EPDS scores for women who had experienced childhood abuse $\left(\mathrm{F}_{(1,39)}=0.571\right.$, $\left.\mathrm{p}>0.05\right)$ in comparison to those who reported not experiencing childhood abuse.

\subsection{Intervention Method and Outcome}

For those women who were identified as being at risk were either referred to the PIMHS team or to other professionals such as their GP's, private psychiatrists and women's health services. Those who received the intervention were asked to complete the EDS questionnaire again to evaluate any change in the depressive symptoms following intervention. In $71.8 \%$ of cases, the second EDS score was lower while in $10.3 \%$ it did not change. A paired t-test indicated that there was not a significant difference between initial EPDS scores and follow up scores $\left(\mathrm{t}_{(14)}=1.115, \mathrm{p}>0.05\right)$.

Following the intervention, a decision about discharging the patient from the PIMHS service was made by the health professional based on the recommendation made by the team during a three monthly review meeting and in discussion with the client. Although EPDS scores are considered, decisions regarding discharge are not made on the basis of EPDS scores alone. Discharged patients are referred for ongoing follow up/treatment with appropriate community and private services.

Based on cross tab analyses it appears that approximately one fifth of the participants (21\%) did not continue with the treatment program offered (i.e. not contactable, or missing a few consecutive appointments) and their case files were closed without any intervention. On evaluating the reasons for termination of treatment before patients were clinically ready to be discharged, the main theme that emerged was the failure of patients to attend appointments or being not contactable. When patients failed to attend the appointment their case manager would make contact with them to check whether they wish to continue with the service. Those patients at the highest level of psychosocial risk are followed up by the Home Visiting service. Those who did not wish to continue with the services but were deemed to be having minimal psychosocial risk and who were not at risk were encouraged to attend the GP services and given a chance to contact PIMHS if required. For those patients whose the contact details had changed or and/or could not be contacted, they were designated as lost for follow up. However, since all other professionals within the hospital including midwives have access to the electronic notes, it would be possible to identify these patients if they presented to any of the other clinics and follow up offered through Home Visiting Service if they continued to have high level of psychosocial risk.

According to the electronic medical records, the duration of treatment by the PIMHS team varied from months to years. For the purpose of this study, length of treatment (duration of accessing intervention from PIMHS service) was categorized as $0<1$ year; $1<2$ years and $>2$ years. Findings revealed that around $80 \%$ of women who received treatment services, received services for less than a year; $9.4 \%$ for 1 to 2 years and $10.4 \%$ used the services for more than 2 years.

\section{Discussion}

Evaluation is an essential part of public health; without evaluation's close ties to program implementation, we may go on using assessment and intervention programs which are ineffective and may have no benefit.

The current screening tools do identify those at risk of or experiencing perinatal depression.

The current study suggests the strongest predictors of postpartum depression are depression or anxiety during pregnancy or having a past history of depressive illness, recent experience of a stressful life event and low levels of social support [6] [32]. Our results are somewhat in line with this with data showing that women with a previous history of depression were more likely to score higher within the clinical risk range on their initial EPDS. More specifically, over half of the women who scored above the risk threshold of 10 on the EPDS had been di- 
agnosed with depression or comorbid anxiety/depression and had received some form of treatment for a mental health issue previously. Our results showed that although a large proportion of women screened scored within the clinical range on the EPDS, most of these women (regardless of whether their pregnancy was planned); felt that they had good support from their partner/family. For these women, it is anticipated that the perceived family/partner support would be protective. Moreover, those who had been identified as being at risk, and had received some intervention showed a slight improvement in their follow up EPDS scores (although not significant).

In gathering the data for this study, we found that collaboration between midwives at the antenatal clinic and PIMHS staff is an effective way of optimising the care of mothers at risk of perinatal depression and this has implications for service development.

\section{Challenges to Clinicians}

Despite the apparent advantage of screening and intervention options, many women seemed to decline or drop out of intervention services. Other studies e.g. [33] [34] (Whitton et al., 1996; McIntosh, 1993) have also noted an unwillingness to be followed up or be involved with ongoing services as a barrier to effective postnatal intervention. Bruits [34] p-13-14 L 39 suggested that other barriers to the access of services include women's beliefs, attitudes and perceptions of mental health. A report by parenting club Bounty in the UK found that a combination of a lack of awareness of the symptoms of postnatal depression and fear of the stigma attached to it were the main reasons preventing thousands of new mothers from seeking help at the early stages of this illness [11]. This may be particularly the case in a culturally diverse area such as the one in which this study is based. In our sample, there were many patients from Cultural and Linguistically Diverse (CALD) backgrounds with 56\% identifying that English is not their first language. Although the service has provisions for accessing the interpreter, services, it is possible that language barriers and reluctance to use interpreters may have contributed to non-attendance at follow up and this will merit further investigation at follow up. Furthermore, researchers have highlighted that there is a high level of stigma, fear/shame associated with mental health diagnoses and thus associated services [35]. Preliminary feedback from PIMHS clinicians, cultural factors seem to play a critical role in determining mental health service use, and the feasibility of the screening instruments employed by the service.

\section{Strength and Limitations}

This is the first systemic auditing of services provided in this area health service.

Given the retrospective nature of this study, one of the main limitations in this study was the frequency of incomplete and inadequate electronic information for review was an issue. As such data including the provision of postnatal interventions and outcomes could not be fully evaluated. Moreover, although in some cases there may have been sufficient information on the initial "book in" assessment including documentation of the EPDS score, subsequent assessments and factors associated with non-attendance were not routinely documented on the electronic databases. As a result the analysis of data is limited to a smaller sample and also a sample that is dependent on having somewhat complete records. This limitation raises questions about routine clinical data collection and record keeping.

\section{Clinical Implications and Recommendations}

The result of these alerts health care providers to be vigilant for those mothers whose pregnancy was unplanned and have history of depression and/or anxiety. Furthermore, family planning strategies, psycho education about mood symptoms and support that meet the needs of "all” including those from CALD, is vital to ensure that women are adequately prepared for pregnancies. This may decrease anxiety and depressive symptoms and result in better pregnancy experience and outcomes. Improve community awareness, including expectant and new mothers about perinatal depression and anxiety. Workforce training (including GP, community nurses and allied health professionals) on perinatal depression, as well as better screening and follow up interventions are all critical.

\section{Future Research}

Further research is needed to examine the needs of women at risk of depression and anxiety during the perinatal period and future research is also required to increase our understanding of the factors that act as barriers or fa- 
cilitators of engagement with mental health services (postnatally), particularly for populations from CALD backgrounds. In addition, research into the development of culturally sensitive interventions to improve perinatal mental health outcomes is vital in communities with culturally diverse populations.

\section{Conclusion}

Standard perinatal care in most places focuses on signs and symptoms of depression in the postpartum period but early identification, appropriate intervention and care coordination during pregnancy are of paramount importance in order to improve mental health outcome of women and their families. Our findings suggest that comprehensive initial assessment at the "book in" clinic including the use of EPDS and detailed psychosocial assessment can help in the early identification of psychosocial and mental health problems, particularly depression. In these cases, appropriate support and intervention can result in quick improvement in clinical symptoms or provide an opportunity for women and their families to gain extra support from the health service. Some consideration has to be made however regarding women from CALD backgrounds. It is suggested that culturally sensitive intervention options are required as a normal course of business as there may be a higher risk of these women failing to attend mainstream health services for emotional symptoms including stress, anxiety and depression. In such instances more efficient use of home visiting services as well as better collaboration between community sectors that cater for women from ethnic minority and CALD backgrounds and mental health staff in hospital would help reduce dropout rates and improve mental health outcomes. Based on our finding that one in five women in our sample were not availing the recommended services, there is an urgent need to identify barriers and facilitators of mental health service use. Addressing this important issue of failure to attend clinical services would help prevent women from suffering in "silence and alone" and may have wider benefits for family and friends.

\section{Acknowledgements}

The authors would like to acknowledge the contributions of the Perinatal and Infant Mental Health Service (PIMHS) team for the clinical work that formed the basis of this report.

\section{Authors Disclosure Statement}

Authors report no conflict of interest. The authors alone are responsible for the content of this manuscript. Authors have no commercial associations that might create a conflict of interest in connection to the work under consideration for publication.

No funding received.

\section{References}

[1] Marcus, M.M., et al. (2012) Depression. A Global Public Health Concern. WHO Department of Mental Health and Substance Abuse. http://dx.doi.org/10.1037/e517532013-004

[2] Institute, B.D. (2012) Depression during Pregnancy \& the Postnatal Period. http://www.blackdoginstitute.org.au/docs/DepressionduringPregnancyandthePostnatalPeriod.pdf

[3] World Health Organisation (2008) Improving Maternal Mental Health: Millennium Developmental Goal 5. http://www.who.int/mental_health/prevention/suicide/Perinatal_depression_mmh_final.pdf

[4] Eberhard-Gran, M., et al. (2004) Depression during Pregnancy and after Delivery: A Repeated Measurement Study. Journal of Psychosomatic Obstetrics \& Gynecology, 25, 15-21. http://dx.doi.org/10.1080/01674820410001737405

[5] Gotlib, I.H., et al. (1989) Prevalence Rates and Demographic Characteristics Associated with Depression in Pregnancy and the Postpartum. Journal of Consulting and Clinical Psychology, 57, 269-274. http://dx.doi.org/10.1037/0022-006X.57.2.269

[6] Moher, D., et al. (2009) Preferred Reporting Items for Systematic Reviews and Meta-Analysis. The PRISMA Statement. PLOS Medicine, 6, e1000097. http://dx.doi.org/10.1371/journal.pmed.1000097

[7] Matthey, S., et al. (2000) Paternal and Maternal Depressed Mood during the Transition to Parenthood. Journal of Affective Disorders, 60, 75-85. http://dx.doi.org/10.1016/S0165-0327(99)00159-7

[8] Shakeel, N., et al. (2015) A Prospective Cohort Study of Depression in Pregnancy, Prevalence and Risk Factors in a Multi-Ethnic Population. BMC Pregnancy Childbirth, 15, 5. http://dx.doi.org/10.1186/s12884-014-0420-0 
[9] Banti, S., et al. (2011) From the Third Month of Pregnancy to 1 Year Postpartum. Prevalence, Incidence, Recurrence, and New Onset of Depression. Results from the Perinatal Depression-Research \& Screening Unit Study. Comprehensive Psychiatry, 52, 343-351. http://dx.doi.org/10.1016/j.comppsych.2010.08.003

[10] Karlsson, L., Melartin, T. and Karlsson, H. (2007) Childhood Stress a Threat to Health in Adulthood. Finnish Medical Journal, 62, 3293-3299.

[11] 4Children (2011) 70,000 Reasons Why Help with Postnatal Depression Has to Be Better. 4Children, UK. http://www.4children.org.uk/Resources/Detail/Suffering-in-Silence

[12] Earls, M.F. (2010) Incorporating Recognition and Management of Perinatal and Postpartum Depression into Pediatric Practice. Pediatrics, 126, 1032-1039. http://dx.doi.org/10.1542/peds.2010-2348

[13] Gunnar, M. and Quevedo, K. (2007) The Neurobiology of Stress and Development. Annual Review of Psychology, 58, 145-173. http://dx.doi.org/10.1146/annurev.psych.58.110405.085605

[14] Segre, L.S., et al. (2011) Disseminating Perinatal Depression Screening as a Public Health Initiative: A Train-TheTrainer Approach. Maternal and Child Health Journal, 15, 814-821. http://dx.doi.org/10.1007/s10995-010-0644-1

[15] Edhborg, M., et al. (2001) The Long-Term Impact of Postnatal Depressed Mood on Mother-Child Interaction: A Preliminary Study. Journal of Reproductive and Infant Psychology, 19, 61-71. http://dx.doi.org/10.1080/02646830123255

[16] Lefkovics, E., Baji, I. and Rigó, J. (2014) Impact of Maternal Depression on Pregnancies and on Early Attachment. Infant Mental Health Journal, 35, 354-365. http://dx.doi.org/10.1002/imhj.21450

[17] Bågedahl-Strindlund, M. and Monsen Börjesson, K. (1998) Postnatal Depression: A Hidden Illness. Acta Psychiatrica Scandinavica, 98, 272-275. http://dx.doi.org/10.1111/j.1600-0447.1998.tb10083.x

[18] Lusskin, S., Pundiak, T. and Habib, S. (2007) Perinatal Depression: Hiding in Plain Sight. The Canadian Journal of Psychiatry, 52, 479-488.

[19] Department of Health, N.Y. (2012) Perinatal Depression.

[20] Leight, K.L., Fitelson, E.M., Weston, C.A. and Wisner, K.L. (2010) Childbirth and Mental Disorders. International Review of Psychiatry, 22, 453-471. http://dx.doi.org/10.3109/09540261.2010.514600

[21] Allen, G. (2011) Early Intervention: The Next Steps, an Independent Report to Her Majesty’s Government by Graham Allen MP. The Stationery Office.

[22] Blue, B. (2008) The National Depression Initiative. Perinatal Mental Health Consortium. Perinatal Mental Health National Action Plan 2008-2010. Full Report.

www.beyondblue.org.au/docs/default-source/8.-perinatal-documents/bw0126-report-beyondblues-perinatal-mental-hea lth-(nap)-summary-report.pdf?sfvrsn=2

[23] Cox, J.L., Holden, J.M. and Sagovsky, R. (1987) Detection of Postnatal Depression: Development of the 10-Item Edinburgh Postnatal Depression Scale. The British Journal of Psychiatry, 150, 782-786. http://dx.doi.org/10.1192/bjp.150.6.782

[24] Kozinszky, Z. and Dudas, R.B. (2015) Validation Studies of the Edinburgh Postnatal Depression Scale for the Antenatal Period. Journal of Affective Disorders, 176, 95-105. http://dx.doi.org/10.1016/j.jad.2015.01.044

[25] Department of Health, G.o.W.A. (2006) Edinburgh Postnatal Depression Scale (EPDS): Translated Versions-Validated. S.P.M.H.R. Group, Ed., 271.

http://www.rikshandboken-bhv.se/Dokument/Edingburgh\%20Depression\%20Scale\%20Translated\%20Gov\%20Wester n\%20Australia\%20Dept\%20Health.pdf

[26] School of Health and Social Development, D.U. (2005) Planning for Effective Health Promotion Evaluation.

[27] Health, N.S.W.D.o. (2010) NSW Health/Families NSW Supporting Families Early Package-SAFE START Guidelines: Improving Mental Health Outcomes for Parents \& Infants. www.health.nsw.gov.au

[28] Matthey, S., et al. (2004) Routine Psychosocial Assessment of Women in the Antenatal Period: Frequency of Risk Factors and Implications for Clinical Services. Archives of Women’s Mental Health, 7, 223-229. http://dx.doi.org/10.1007/s00737-004-0064-6

[29] Eberhard-Gran, M., Slinning, K. and Rognerud, M. (2014) Screening for Postnatal Depression-A Summary of Current Knowledge. Tidsskrift for den Norske Legeforening, 134, 297-301.

[30] Murray, L. and Carothers, A.D. (1990) The Validation of the Edinburgh Post-Natal Depression Scale on a Community Sample. The British Journal of Psychiatry, 157, 288-290. http://dx.doi.org/10.1192/bjp.157.2.288

[31] Boyce, P., Stubbs, J. and Todd, A. (1993) The Edinburgh Postnatal Depression Scale: Validation for an Australian Sample. Australian and New Zealand Journal of Psychiatry, 27, 472-476. http://dx.doi.org/10.3109/00048679309075805

[32] Andersson, L., Sundström-Poromaa, I., Wulff, M., Åström, M. and Bixo, M. (2006) Depression and Anxiety during 
Pregnancy and Six Months Postpartum: A Follow-Up Study. Acta Obstetricia et Gynecologica Scandinavica, 85, 937944. http://dx.doi.org/10.1080/00016340600697652

[33] Whitton, A., Warner, R. and Appleby, L. (1996) The Pathway to Care in Post-Natal Depression: Women’s Attitudes to Post-Natal Depression and Its Treatment. British Journal of General Practice, 46, 427-428.

[34] Buist, A., et al. (2005) Recognition and Management of Perinatal Depression in General Practice: A Survey of GPs and Postnatal Women. Australian Family Physician, 34, 787-790.

[35] Blignault, I., Smith, S., Woodland, L., Ponzio, V., Ristevski, D. and Kirov, S. (2010) Fear and Shame: Using Theatre to Destigmatise Mental Health Illness in an Australian Macedonian Community. Health Promotion Journal of Australia, 21, 120-126.

\section{Submit or recommend next manuscript to SCIRP and we will provide best service for you:}

Accepting pre-submission inquiries through Email, Facebook, Linkedin, Twitter, etc A wide selection of journals (inclusive of 9 subjects, more than 200 journals)

Providing a 24-hour high-quality service

User-friendly online submission system

Fair and swift peer-review system

Efficient typesetting and proofreading procedure

Display of the result of downloads and visits, as well as the number of cited articles

Maximum dissemination of your research work

Submit your manuscript at: http://papersubmission.scirp.org/ 